\title{
Sphingolipid biology and its role in cancer development and therapy
}

\author{
Alejandro Carvajal ${ }^{1}$, Alvaro Menendez ${ }^{1}$, Wayne Bowen ${ }^{2}$ and Harold Wanebo ${ }^{2,3}$ \\ ${ }^{1}$ Department of Medicine, Roger Williams Medical Center - Boston University School of Medicine, USA \\ ${ }^{2}$ Department of Biology, Pharmacology, Brown University, Providence, RI, USA \\ ${ }^{3}$ Department of Surgery, Boston University School of Medicine, Boston, MA, USA
}

\section{Introduction}

Sphingolipids are important molecules involved in controlling various aspects of normal cell functioning. The objective of this review is to explore the elements of sphingolipid metabolism and connect its features to cancer biology and development as well as its role in current and potential treatments. Due to their wide range of action and functioning on cells, sphingolipids have been the target of research on the role they might play on cancer biology as well as their role in therapeutics against cancer. In this review article, we discuss the primary components in sphingolipid metabolism, as well as their role in cancer development, chemotherapy and radiation resistance, prognostics, and current and potential treatments. We use previously published research ranging from molecular biology journals to clinical oncology journals in order to broaden the scope of review. Our hope is to reach out to and educate both basic researchers as well as practicing clinicians on sphingolipid biology and its role in current and potential treatments for cancer.

\section{Ceramide}

\section{Structure/Metabolism - regulation of ceramide metabolism}

Sphingolipids, while once thought to only play a structural role in cell membrane function, are now typically described as effector molecules which are involved in controlling various aspects of normal cell growth, proliferation, inhibition, apoptosis induction, senescence, cell migration, and inflammation. Ceramide acts as a central molecule of sphingolipid metabolism, as it is involved in sphingolipid synthesis and catabolism. Ceramide is composed of a sphingosine base and amidelinked acyl chains varying in length from $\mathrm{C}_{14}$ to $\mathrm{C}_{26}$. Ceramide can be generated by several separate mechanisms. The first is the generation via sphingomyelinases (SMases), which hydrolyze sphingomyelin (SM) to yield ceramide. The second mechanism involves formation of endogenous ceramide via a de novo pathway. This pathway requires condensation of serine and palmitoyl CoA, leading to synthesis of dihydroceramide by dihydroceramide synthases. Dihydroceramide is then converted to ceramide via desaturase. The third mechanism is via the "salvage pathway". In this pathway, sphingosine serves as the product of sphingolipid catabolism, and is salvaged through reacylation, resulting in the generation of ceramide or its derivatives (Figure 1). This is the same mechanism by which exogenous ceramide is recycled, as it is broken down into sphingosine, which is then generated into ceramide by ceramide synthase.

Once ceramide is formed, it can be used as a substrate by ceramidases to free sphingosine, which can be further metabolized into sphingosine-1-phosphate (S1P). Ceramide can also be metabolized into glucosylceramide or sphingomyelin in the Golgi.

\section{Ceramide signaling in cancer - Role in pathogenesis and drug resistance}

Ceramide has been found to effect pathogenesis in not only normal cell functioning, but in cancer as well. Apoptosis and autophagy are two important functions by which ceramide is involved in. Apoptosis is programmed cell death which is essential for proper development and the maintenance of cell homeostasis. It is initiated via an extrinsic or intrinsic pathway. Autophagy is a catabolic process in which cytoplasmic components are sequestered in autophagosomes, and delivered to lysosomes for degradation and recycling. Autophagy promotes cell survival during periods of stress, including hypoxia or nutrient deprivation, however autophagy can also mediate cell death.

While ceramide was first recognized as a regulator for apoptosis in the 1990s, it has more recently been implicated to play a role in the induction of autophagy. While the mechanism by which ceramide induces autophagy is unclear, there are several methods of treatment by which ceramide has been found to induce autophagy. One study done showed that treatment of human colon cancer and breast cancer cell lines with exogenous C2-ceramide, tamoxifen, or 1-phenyl2-palmitoylamino-3-morpholino-1-propanol (PDMP) result in accumulation of ceramide, thus suppressing Akt, which is a molecule that is normally involved in inhibiting autophagy [1]. Additionally, the same study found that C2-ceramide, tamoxifen, and PDMP enhanced the expression of Beclin 1. Increased expression of Beclin 1 has been found to be a contributing factor in autophagy in human leukemia cell lines treated with arsenic trioxide [2]. In malignant glioma cell lines treated with C2-ceramide, there is enhanced expression of BNIP3, a protein which promotes Beclin 1 dissociation from its inhibitory complex, leading to autophagic cell death [3].

As important as ceramide and its metabolites have been shown to be in potential treatments of cancer, its pathogenesis is crucial to understand as it is also involved in some drug-resistant aspects of cell

Correspondence to: Alejandro Carvajal, Department of Medicine, Roger Williams Medical Center - Boston University School of Medicine, 19 Joy Street, Providence, RI 02908, USA, Tel: 617-780-0458; E-mail: Carv108@Gmail.com

Received: February 02, 2016; Accepted: February 29, 2016; Published: March 04,2016 


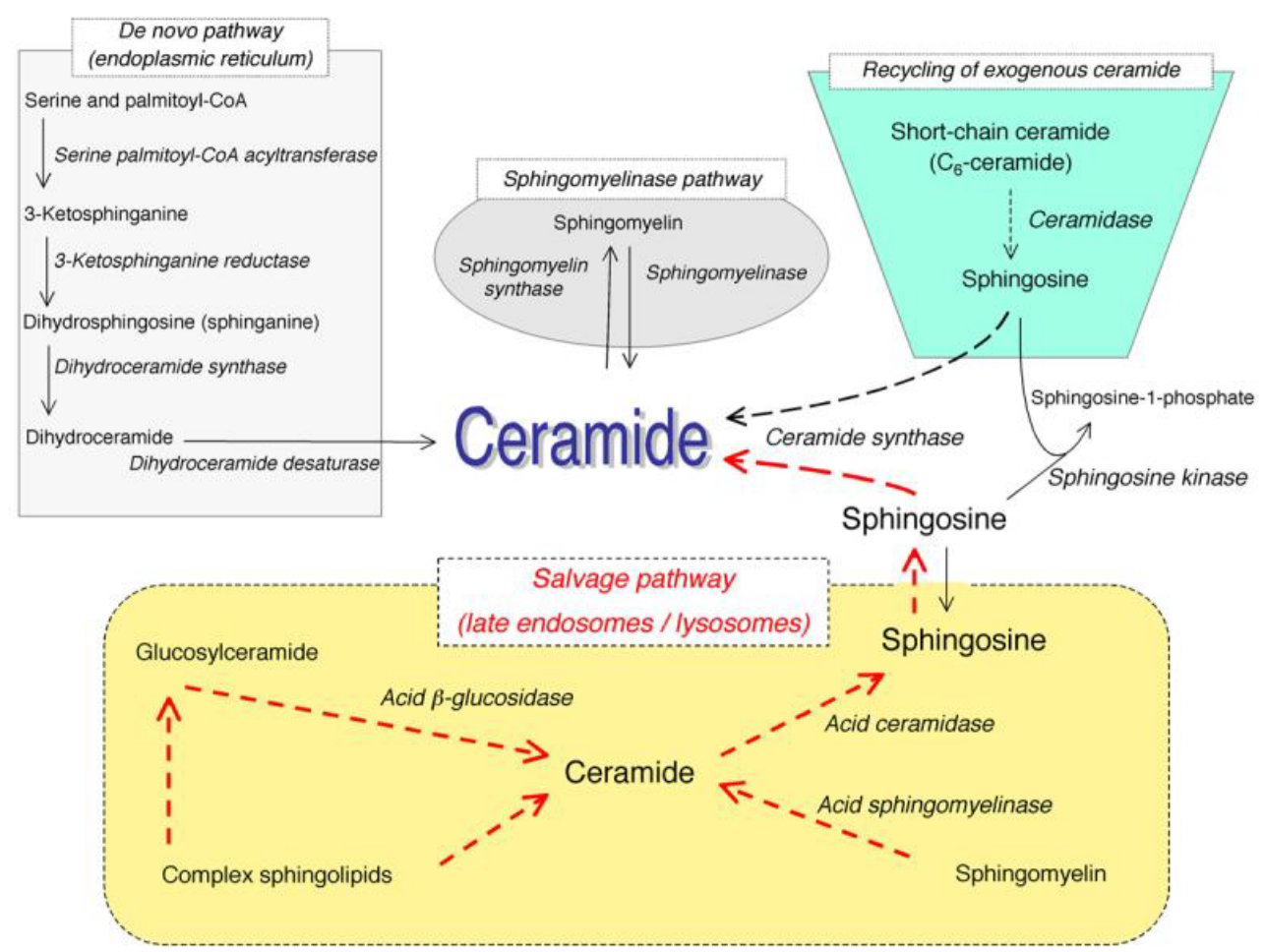

Figure 1. Pathways of ceramide synthesis.

The above figure illustrates the several different pathways with which ceramide can be synthesized. These include the sphingomyelinase pathway, the de novo pathway, the exogenous ceramide-recycling pathway, and the salvage pathway. (Kitatani K, et al. The sphingolipid salvage pathway in ceramide metabolism and signaling. Cell Signal. 2008 June; 20(6):1010-8. doi: 10.1016/j.cellsig.2007.12.006. Epub 2007 Dec 14).

functioning. Some studies have shown that one of the mechanisms of resistance that cancer cells develop against chemotherapy is the alteration of ceramide accumulation. Since ceramide is rapidly metabolized by glucosylceramide synthase (GCS), the cancer cells have developed a way to increase expression of this enzyme [4]. Sphingosine1-phosphate (S1P) and sphingosine kinase 1 (SK1) have been shown to promote proliferation and prevent drug-induced apoptosis. Elevated expression of SK1 and the increased levels of S1P have been observed in many types of cancer, such as colon, breast, uterus, kidney, and lung, among others [5]. S1P is generated from ceramide, and requires SK1. S1P has mitogenic effects, both as an extracellular signaling molecule and as an intracellular second messenger. S1P can initiate downstream $G$ protein mediated signaling pathways, leading to a variety of responses including cell proliferation, inhibition of apoptosis and cell migration. S1P can also exert its proliferative and survival effects by activating a known anti-apoptotic transcription factor, NF- $\kappa B$ [6]. Finally, S1P can prevent ceramide-induced mitochondrial events including cytochrome c release and caspase activation [7].

\section{Ceramide-based anti-cancer therapeutics}

The use of sphingolipid-based anti-cancer therapeutics present a promising option for treating cancer in the future. There have been several methods proposed and tested on this front. The use of ceramide analogues have been shown to promote apoptotic/ autophagic pathways in cancer cells. Liposome-mediated delivery of C6-ceramide has been shown to be an effective drug delivery method. Use of liposomal C6-ceramide targets nuclear accumulation in MDA human breast cancer cells. Moreover these targeted C6- ceramides can regulate Akt phosphorylation and activate caspases to induce apoptosis [8]. Additionally, the PEGylated form of C6-ceramide encapsulated in liposomes has been shown to be effective against murine breast adenocarcinoma models [9].

In studies done at Brown University, the unique anti-cancer activity of permeable C6-ceramide was demonstrated by significantly increasing apoptosis in aggressive pancreatic cancer cell lines (L3.6, PANC-1, and MIA PACA2) in vitro and significantly enhancing tumor regression and survival in SCID mice bearing L3.6 transplants. C6-ceramide also demonstrated a synergistic effect with cetuximab (an anti-EGFR biologic) in spite of common presence of KRAS mutations, which render them resistant to cetuximab. At the molecular level, C6-ceramide combined with gemcitabine or paclitaxel induced a significant time dependent decrease in P13K/AKT pathway (p-GSK3a/b), TORC1 pathway (p-S6 and P-4E-BPI), and pERK (RAS/MEK/ ERK) pathway in L3.6 pancreatic cancer cells. These effects disrupt the major cell signaling pathway including inhibition of pro-survival (P13K/AKT/mTOR and mutant ERK/MAPK/KRAS) pathways, resulting in enhanced cytotoxicity of gemcitabine and paclitaxel cancer cell cytotoxicity. This data suggests C6-ceramide can inhibit key survival pathways resulting in chemo synergism with gemcitabine, paclitaxel, and cetuximab, both in vitro and in vivo, and can potentially pave the way for development of C6-ceramide as an anti-cancer biologic [10].

An alternative strategy is to induce ceramide formation by inhibiting ceramidase. Inhibition of ceramidase leads to ceramide accumulation, thereby causing apoptotic cell death in cancerous cells. One of the inhibitors used is B13. B13 inhibits acid ceramidase, which has been shown to induce cell death in cultured prostate cancer cells [11]. 


\section{Glucosylceramide synthase}

\section{Ceramide glycosylation and cancer drug resistance}

As stated previously, ceramide can go through several pathways of metabolism, and its products can be used for several different roles. Glucosylceramide (GC) is one of those breakdown products. Ceramide is converted to GC by GCS. Since GCS decreases the amount of ceramide in cells, this indirectly acts to protect cancer cells from chemotherapyinduced apoptosis. This has been seen in several different cancer cell types, especially breast cancer cells [12]. Additionally, it has been shown that glucosylceramide accumulates in Adriamycin-resistant breast carcinoma cells, in vinblastine-resistant epitheliod carcinoma cells, and in tumor specimens from patients showing poor response to chemotherapy [13].

\section{Targeting ceramide glycosylation to reverse drug resistance (ASM)}

There have been several methods for targeting ceramide glycosylation to reverse drug resistance. Use of GCS inhibitors has revealed how decreases in cellular levels of neutral glycosphingolipids and gangliosides, and elevations in ceramide, causes cell cycle arrest [14]. While there are no currently approved regimens, there has been significant research dedicated towards this pathway. For example, combinations of fenretinide (4-HPR), which is known to elevate ceramide and dihydroceramide, with inhibitors of GCS or SK, such as PDMP, were reported to synergistically suppress the growth of various cancer cells [15]. PDMP has also been used on human colon cancer and breast cell lines. It was shown to increase ceramide levels and stimulate autophagy in these cell lines [16].

\section{Use of acid sphingomyelinase in cancer therapy}

\section{Use of acid sphingomyelinase as adjunct to cancer therapy}

Sphingomyelin can be converted into ceramide via the action of sphingomyelinase (SMase). There are several different SMases characterized by the $\mathrm{pH}$ required for optimal enzymatic activity. While acid sphingomyelinase (ASM) is best known for its involvement in the lysosomal storage disorder Niemann-Pick disease (NPD), it has more recently been implicated in cancer biology. In an analysis of the microarray database Oncomine (www.oncomine.org, January 3, 2008), it was revealed that $12 / 104$ matched cancer $v$ s. normal tissue comparisons under expressed the ASM mRNA. This would predict that in at least some cancers ASM may be down-regulated, contributing to their reduced ceramide content and perhaps directing the cells away from apoptosis and toward proliferation [17]. It has been shown that ionizing radiation causes cell death via SMase-mediated production of ceramide, which in turn leads to apoptosis of the cell. In a study done by researchers using ASM knockout mice as well as lymphoblasts from patients with NPD, it was found that these cells failed to respond to ionizing radiation with generation of ceramide and apoptosis [18]. The use of recombinant ASM has already been produced and is being evaluated for the treatment of NPD, and its use as an adjunct to cancer therapy is now being examined. Recombinant ASM is known to be hepatotropic in nature; because of this, it was used in a study to evaluate it as an adjuvant treatment with sorafenib in experimental models of hepatocellular carcinoma. The combination therapy exhibited a synergistic effect on reducing tumor volume and blood vessel density in the effect cells. However, there was not any increase in survival from the recombinant ASM/sorafenib treatment, and the study concluded that the results merited further investigation [19]. In another study, it was demonstrated that ASM levels of expression actually determine the malignant phenotype of melanoma cells in terms of pigmentation, tumor progression, invasiveness and metastatic ability [20].

\section{Hypoxia-resistance and sphingosine phosphate in cancer therapy}

\section{Hypoxia inducible factor-family factors - regulation /role in cancer therapy}

Hypoxia occurs when there is decreased oxygenation to a cell. Hypoxia is known to be an important process in solid tumors, and is a poor prognostic factor for response to therapeutics and survival of patients. As a tumor develops, the diffusion distance from the existing vasculature increases resulting in hypoxia, which in turn drives the overexpression of angiogenic factors such as VEGF, leading to the formation of a new vasculature in an attempt to provide adequate supply of oxygen to the tumor. Hypoxia-inducible factors (HIFs) are transcription factors that respond to changes in available oxygen in the cellular environment. HIF-1 $\alpha$ is a specific factor that has been specifically focused on in cancer research. HIF-1a responds rapidly to states of decreased oxygenation by regulating genes that promote neoangiogenesis, glycolysis, and increased tumor growth, all of which are essential for tumor growth. In one study, HIF-1a was found to be overexpressed in 13 of 19 tumor types compared with the respective normal tissues, including colon, breast, gastric, lung, skin, ovarian, pancreatic, prostate, and renal carcinomas [21]. Many of the novel anticancer drugs work via the targeting of HIF-1 $\alpha$. These drugs include BCR-ABL inhibitor imatinib/Gleevec, epidermal growth factor receptor inhibitors gefitinib/Iressa, erlotinib/Tarceva and cetuximab/ C225, and HER2 $2^{\text {neu }}$ inhibitor trastuzumab/Herceptin. Radiation has been shown to induce HIF-1 $\alpha$ activity, leading to the production of VEGF and other angiogenic cytokines that protect the endothelial cells of the tumor vasculature from radiation-induced death [22]. Therefore, by inhibiting HIF-1 $\alpha$, one can expect to see dramatically increased radiation-induced vessel destruction and tumor control.

\section{Role of sphingosine phosphate signaling as a target for anti- hypoxia therapy}

Sphingosine-1-phosphate (S1P) is a product of ceramide metabolism, and it has been shown to lead to cancer cell proliferation and antiapoptosis [23].S1P is generated from sphingosine, in a reaction catalyzed by sphingosine kinase 1 (SK1). In numerous studies, SK1/ S1P have been shown to be tumor-promoting molecules, and elevated levels of these molecules have been observed in different cancer and tumor tissues. More specifically, evidence has linked these molecules with adaptation of cancer cells to hypoxia. In a study conducted with five distinct tumor models, SK1 was shown to be rapidly stimulated under hypoxic conditions. This interaction appeared to be dependent on the generation of reactive oxygen species (ROS) production [24]. In another study, it was found that in glioblastomas, increased vascularity and increased SK1 activity was correlated to aggressive behavior and poor survival in patients. In prostate cancer, the study showed that SK1 activity was strikingly increased in tumor samples and correlated with prostate-specific antigen level and tumor grade. Given the importance of HIF-1a stimulation in solid tumor neoangiogenesis and metastasis, a wide range of pharmacological approaches have been proposed including the targeting of the SK1/S1P signaling. A recent study has applied the use of an anti-S1P monoclonal antibody called sphingomab. The study found that inhibiting extracellular S1P signaling resulted in blockage of HIF-1a accumulation and activity in several cancer cell 
models exposed to hypoxia. Additionally, the study demonstrated that administration of the antibody for 5 days before chemotherapy is more effective at local tumor control and metastatic dissemination than any other treatment scheduling [25]. Another well-explored therapeutic is sonepcizumab. Sonepcizumab is a humanized monocloncal antibody to S1P that acts as a molecular sponge to reduce S1P signaling which, in nonclinical models, inhibited tumor growth. In a phase I clinical trial, sonepcizumab was well tolerated in 23 patients with advance tumors. Phase II trials are planned [26].

\section{Sphingolipid Metabolism and its role in cancer}

\section{Sphingosine kinase - regulatory of sphingolipid signals and role in cancer}

Sphingosine kinase 1 (SK1) is an essential enzyme in the sphingolipid pathway that regulates the relative levels of S1P, sphingosine, and ceramide. While ceramide and sphingosine have been established as antigrowth molecules, S1P offers a progrowth message to cells. Elevated levels (around $\sim 2-3$ folds increases) in SK1 expression have been detected in many human cancers, such as breast, lung, prostate and colon cancer [27].SK1 promotes cancer cell survival and tumorigenesis, as studies have shown that various hormones, cytokines, and growth factors cause a rapid activation of SK1, and that inhibition of SK1 in turn leads to inhibition of cell growth. Not only does SK1 help promote tumor growth, but it also has an important role in prevention of apoptosis (Figure 2). This can be seen particularly in tumor cell lines in response to apoptotic stimuli, such as TNF, Fas ligand, serum deprivation, ionizing radiation and anti-cancer drugs. Increased SK1 activity has been shown to contribute to cancer cells being resistant to ionizing radiation and anticancer drugs. Although the precise mechanisms remain to be identified, SK1 appears to affect apoptosis via both the intrinsic and extrinsic pathways $[28,29]$.

\section{Sphingosine kinase inhibiton - therapeutic potential}

Given the oncogenic properties of SK1, use of an SK inhibitor offers to be an exciting potential therapeutic agent in certain cancers. One of the molecular inhibitors of SK1 that has been published has been effective in mice in slowing the growth of murine mammary carcinoma without any apparent toxicity [30]. SK2 has also been a target for SK inhibitor therapy. A novel SK2 inhibitor, ABC294640, was shown to induce non-apoptotic death in kidney carcinoma cells as well

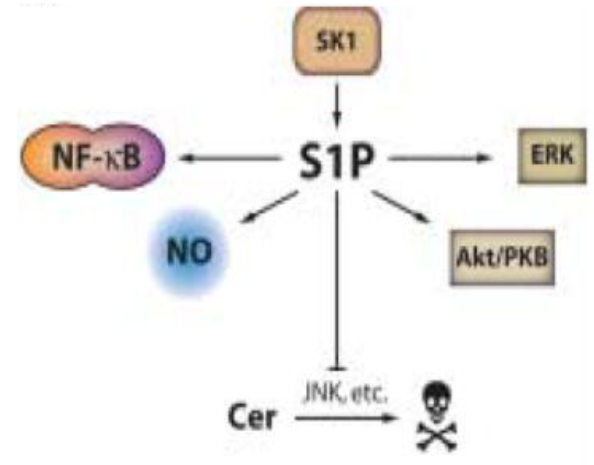

Figure 2. Pro-oncogenic and antiapoptotic effects of SK1 and S1P.

SK1 increases levels of S1P. S1P encourages proliferation of cells by activating prosurvival mediators like Akt, nitric oxide, and extracellular signal-regulated kinase (ERK). It also inhibits apoptosis by activating known anti-apoptotic transcription factor $\mathrm{NF}-\mathrm{\kappa B}$, and inhibiting ceramide-mediated apoptosis. (Taha TA, et al. A house divided: ceramide, sphingosine, and sphingosine-1-phosphate in programmed cell death. Biochim Biophys Acta. 2006 Dec;1758(12):2027-36. Epub 2006 Nov 1). as autophagic responses in prostate and breast adenocarcinoma cells [31]. It has also been found that SK2 was overexpressed in multiple myeloma cell lines. Application of ABC294640 was shown to effectively limit myeloma cell line proliferation and induced caspase 3-mediated apoptosis. It was additionally found to act synergistically with a bcl2 inhibitor in inducing myeloma cell death [32]. Another type of sphingosine kinase inhibitor is FTY720. FTY720 is a sphingosine-based immunosuppressant, initially showed to have successful outcomes against multiple sclerosis. It has also been shown to inhibit ceramide synthesis via ceramide synthase. It does this via induction of caspaseindependent cell death through the downregulation of nutrient transporters and induction of autophagy [33]. Pharmacologic doses of FTY720 were shown to induce apoptosis and impair clonogenicity in blast crises in chronic myelogenous leukemia and Philadelphia chromosome-positive acute lymphocytic leukemia. More recently, advances have been made with sphingosine kinase inhibition. Safingol, an inhibitor of SKI, has been studied in a phase I clinical trial. In the trial, safingol was given alone and in combination with cisplatin in 43 patients with advanced solid tumors. The study, which is now ready for phase II, concluded that safingol can safely be administered with cisplatin [34].

\section{Alpha-galactosylceramides and modifications - role in cancer therapy}

Natural killer T (NKT) cells are a heterogeneous group of $\mathrm{T}$ cells that share properties of both $\mathrm{T}$ cells and natural killer cells. NKT cells exert strong anti-tumor activity in vivo and in vitro, which has made the subject of research for potential cancer therapy. NKT cells are activated by a specific glycolipid antigen, $\alpha$-galactosylceramide, which has made it the target of several clinical trials. In one such trial, a-galactosylceramide-pulsed antigen presenting cells were administered in the submucosa of patients with head and neck cancer. This resulted in increased numbers of NKT cells and enhanced natural killer activity in these patients [35]. This method was also shown to be effective by decreasing tumor growth of pancreatic cancer cells in a different trial [36].

\section{Natural products as platforms for design of sphingolipid related anti-cancer agents}

Research has been conducted to determine if there can be benefit from using natural products as anti-cancer agents. Fungal metabolites have been one of the natural products that have been studied. One of the more well-studied fungal metabolites is myriocin, which is isolated from Myriococcum ablomyces, and is a specific inhibitor of serine palmitoyltransferase (SPT). SPT is the enzyme that catalyzes the first step of the biosynthesis of all sphingolipids. Inhibition of SPT leads to a decrease in ceramide, thereby reducing inflammatory responses, and in some cases, causing immunosuppression. In a study done in 2011, myriocin induced growth inhibition in melanoma cells by inducing cell cycle arrest in the G2/M phase. Additionally, expression of antiproliferative genes $\mathrm{p} 53$ and p 21 were increased [37]. In a follow up study, myriocin was administered intradermally or intraperitoneally into melanoma mice. Tumor formation was significantly inhibited in these mice when compared to controls [38]. In another study, it was found that myriocin not only induced death of lung cancer cells via apoptosis, but synergistically inhibited cancer cell growth when given in combination with anti-tumor drugs docetaxel and cisplatin [39].

Jaspine $\mathrm{B}$ is an anhydrophytosphingosine which is a derivative 
isolated from the marine sponge Jaspis sp. Jaspine B works by inhibiting sphingomyelin synthase, the enzyme which converts ceramide in sphingomyelin. This leads to increased concentrations of ceramide within the cell. In one study, Jaspine B was able to dose- and timedependently decrease the viability of murine B16 and human SK-Mel28 melanoma cells via triggering of apoptosis [40]. In another study, the cytotoxicity of jaspines was examined in A549 human alveolar cells. Results of cells culture with Jaspine B revealed significant increases in dihydroceramide and eventual cell death. Only slight increases in ceramide were noted.

\section{Conclusion}

Sphingolipids are a diverse group of lipids which serve a variety of functions in both mammalian development and physiology. While the scope of sphingolipid biology is vast, its role in cancer has been highlighted in this review of literature. Clearly, sphingolipid metabolism is essential in the cell life cycle. Despite the large number of preclinical trials showing promising results, clinical trials utilizing sphingolipids in cancer are sparse. However, the development of recent phase I and upcoming phase II trials brings to life the promising future of the therapeutic potential of sphingolipids in cancer. Understanding sphingolipid metabolism in finer detail will help researchers discover potential new treatments for malignant, and other pathological processes alike. It is our hope that the above review article can help both researchers and clinicians gain a better understanding of sphingolipid metabolism and its applications in cancer medicine, and eventually use it in their daily practice and research.

\section{References}

1. Scarlatti F, Bauvy C, Ventruti A, Sala G, Cluzeaud F, et al. (2004) Ceramide-mediated macroautophagy involves inhibition of protein kinase B and up-regulation of beclin 1 . J Biol Chem 279: 18384-18391.[Crossref]

2. Dbaibo GS, Kfoury Y, Darwiche N, Panjarian S, Kozhaya L, et al. (2007) Arsenic trioxide induces accumulation of cytotoxic levels of ceramide in acute promyelocytic leukemia and adult T0cell leukemia/lymphoma cells through de novo ceramide synthesis and inhibition of glucosylceramide synthase activity. Haematologica 92: 753-762. [Crossref]

3. Daido S, Kanzawa T, Yamamoto A, Takeuchi H, Kondo Y, et al. (2004) Pivotal role of the cell death factor BNIP3 in ceramide-induced autophagic cell death in malignant glioma cells. Cancer Res 64: 4286-4293.[Crossref]

4. Gouazé V, Liu YY, Prickett CS, Yu JY, Giuliano AE, et al. Glucosylceramide synthase blockade downregulates P-glycoprotin and resensitizes multidrug-resistant breast cancer cells to anticancer drugs. Cancer Res 65: 3861-3867. [Crossref]

5. Komarova YA, Mehta D, Malik AB (2007) Dual regulation of endothelial junctional permeability. Sci STKE 2007: re8.[Crossref]

6. Maceyka M, Payne SG, Milstien S, Spiegel S (2002) Sphingosine kinase, sphingosine1-phosphate, and apoptosis. Biochim Biophys Acta 1585: 193-201.[Crossref]

7. Levade T, Augé N, Veldman RJ, Cuvillier O, Nègre-Salvayre A, et al. (2001) Sphingolipid mediators in cardiovascular cell biology and pathology. Circ Res 89: 957968.[Crossref]

8. Stover T, Kester M (2003) Liposomal delivery enhances short-chain ceramide-induced apoptosis of breast cancer cells. J Pharmacol Exp Ther 307: 468-475.[Crossref]

9. Stover TC, Sharma A, Robertson GP, Kester M (2005) Systemic delivery of liposomal short-chain ceramide limits solid tumor growth in murine models of breast adenocarcinoma. Clin Cancer Res 11: 3465-3474.[Crossref]

10. Cao C, Lu S, Shrayer D, Wanebo H, Wan Y, et al.C6-Ceramide enhances the in vitro and in vivo chemo responsiveness of pancreatic cancer cells to Paclitaxel and Gemcitabine by inhibiting pro-survival P13K/AKT/mTOR and ERKK/MAPK (KRAS) Pathway. Manuscript in submission.

11. Samsel L, Zaidel G, Drumgoole HM, Jelovac D, Drachenberg C, et al. (2004) The ceramide analog, B13, induces apoptosis in prostate cancer cell lines and inhibits tumor growth in prostate cancer xenografts. Prostate 58: 382-393.[Crossref]
12. Lucci A, Cho WI, Han TY, Giuliano AE, Morton DL, et al. (1998) Glucosylceramide: a marker for multiple-drug resistant cancers. Anticancer Res 18: 475-480.[Crossref]

13. Rani CS, Abe A, Chang Y, Rosenzweig N, Saltiel AR, et al. (1995) Cell cycle arrest induced by an inhibitor of glucosylceramide synthase.Correlation with cyclindependent kinases. J Biol Chem 270: 2859. [Crossref]

14. Maurer BJ, Metelitsa LS, Seeger RC, Cabot MC, Reynolds CP (1999) Increase of ceramide and induction of mixed apoptosis/necrosis by N-(4-hydroxyphenyl)retinamide in neuroblastoma cell lines. J Natl Cancer Inst 91:1138-1114 [Crossref]

15. Scarlatti F, Bauvy C, Ventruti A, Sala G, Cluzeaud F, et al. (2004) Ceramide-mediated macroautophagy involves inhibition of protein kinase B and up-regulation of beclin 1 . J Biol Chem 279: 18384-18391.[Crossref]

16. Flowers M, Fabriás G, Delgado A, Casas J, Abad JL, et al. (2012) C6-ceramide and targeted inhibition of acid ceramidase induce synergistic decreases in breast cancer cell growth. Breast Cancer Res Treat 133: 447-458.[Crossref]

17. Zhong H, De Marzo AM, Laughner E, Lim M, Hilton DA, et al. (1999) Overexpression of hypoxia-inducible factor 1alpha in common human cancers and their metastases. Cancer Res59: 5830-5835.[Crossref]

18. Smith EL, Schuchman EH (2008) The unexpected role of acid sphingomyelinase in cell death and the pathophysiology of common diseases. FASEB J 22: 3419-3431.[Crossref]

19. Santana P, Peña LA, Haimovitz-Friedman A, Martin S, Green D, et al. (1996) Acid sphingomyelinase-deficient human lymphoblasts and mice are defective in radiationinduced apoptosis. Cell 86: 189-199.[Crossref]

20. Bizzozero L, Cazzato D, Cervia D, Assi E, Simbari F, et al. (2014) Acid sphingomyelinase determines melanoma progression and metastatic behaviour via the microphtalmia-associated transcription factor signalling pathway. Cell Death Differ 21: 507-520. [Crossref]

21. Laughner E, Taghavi P, Chiles K, Mahon PC, Semenza GL (2001) HER2 (neu) signaling increases the rate of hypoxia-inducible factor 1a (HIF-1a) synthesis: novel mechanism for HIF-1-mediated vascular endothelial growth factor expression. $\mathrm{Mol}$ Cell Biol 21: 3995-4004.

22. Zheng W, Kollmeyer J, Symolon H, Momin A, Munter E, et al. (2006) Ceramides and other bioactive sphingolipid backbones in health and disease: lipidomic analysis, metabolism and roles in membrane structure, dynamics, signaling an autophagy. Biochem Biophys Acta 1758:1864-1884. [Crossref]

23. Liu Y, Wada R, Yamashita T, Mi Y, Deng CX, et al. (2000) Edg-1, the G proteincoupled receptor for sphingosine-1-phosphate, is essential for vascular maturation. $J$ Clin Invest 106: 951-961.[Crossref]

24. Melillo G (2006) Inhibiting hypoxia-inducible factor 1 for cancer therapy. Mol Cancer Res4: 601-605.[Crossref]

25. LaMontagne K, Littlewood-Evans A, Schnell C, O’Reilly T, Wyder L, et al. (2006) Antagonism of sphingosine-1-phosphate receptors by FTY720 inhibits angiogenesis and tumor vascularization. Cancer Res 66: 221-231.[Crossref]

26. Gordon MS, Just R, Rosen LS, Dorr A (2010)A phase I study of sonepcizumab, a humanized monoclonal antibody to shingosine-1-phosphate, in patients with advanced tumors. J Clin Oncol 28: 15.

27. Hait NC, Oskeritzian CA, Paugh SW, Milstien S, Spiegel S (2006) Sphingosine kinases, sphingosine 1-phosphate, apoptosis and diseases. Biochim Biophys Acta 1758: 2016-2026. [Crossref]

28. Xia P, Wang L, Gamble JR, Vadas MA (1999) Activation of sphingosine kinase by tumor necrosis factor-alpha inhibits apoptosis in human endothelial cells. $J$ Biol Chem 274: 34499-34505.[Crossref]

29. Limaye V, Li X, Hahn C, Xia P, Berndt MC, et al. (2005) Sphingosine kinase-1 enhances endothelial cell survival through a PECAM-1-dependent activation of PI-3K/ Akt and regulation of Bcl-2 family members. Blood 105: 3169-3177.[Crossref]

30. Cuvillier O, Levade T (2001) Sphingosine 1-phosphate antagonizes apoptosis of human leukemia cells by inhibiting release of cytochrome $\mathrm{c}$ and Smac/DIABLO from mitochondria. Blood 98: 2828-2836.[Crossref]

31. French KJ, Upson JJ, Keller SN, Zhuang Y, Yun JK, et al. (2006) Antitumor activity of sphingosine kinase inhibitors. J Pharmacol Exp Ther 318: 596-603.[Crossref]

32. Venkata JK, An N, Stuart R, Costa LJ, Cai H, et al. (2014) Inhibition of sphingosine kinase 2 downregulates the expression of c-Myc and Mcl-1 and induces apoptosis in multiple myeloma. Blood 124: 1915-1925.[Crossref]

33. Wallington-Beddoe CT, Hewson J, Bradstock KF, Bendall LJ (2011) FTY720 produces 
caspase-independent cell death of acute lymphoblastic leukemia cells. Autophagy 7: 707-715.[Crossref]

34. Dickson MA, Carvajal RD, Merrill AH Jr, Gonen M, Cane LM, et al. (2011) A phase I clinical trial of safingol in combination with cisplatin in advanced solid tumors. Clin Cancer Res 17: 2484-2492.[Crossref]

35. Uchida T, Horiguchi S, Tanaka Y, Yamamoto H, Kunii N, et al. (2008) Phase I study of a-galactosylceramide-pulsed antigen presenting cells administration to the nasal submucosa in unresectable or recurrent head and neck cancer. Cancer Immunol Immunother 57: 347-355.[Crossref]

36. Nagaraj S, Ziske C, Strehl J, Messmer D, Sauerbruch T, et al. (2006) Dendritic cells pulsed with alpha-galactosylceramide induce anti-tumor immunity against pancreatic cancer in vivo. IntImmunol 18: 1279-1283.[Crossref]
37. Smyth MJ, Crowe NY, Pellicci DG, et al. (2002) Sequential production of Lee YS, et al. Serine palmitoyltransferase inhibitor myriocin induces growth inhibition of B16F10 melanoma cells through G(2)/M phase arrest. Cell Prolif 44:320-9.

38. Lee YS1, Choi KM, Lee S, Sin DM, Lim Y, et al. (2012) Myriocin, a serine palmitoyltransferase inhibitor, suppresses tumor growth in a murine melanoma mode by inhibiting de novo sphingolipid synthesis. Cancer Biol Ther 13:92-100. [Crossref]

39. Choi KE, Jung YS, Kim DH, Song JK, Kim JY, et al. (2014) Myriocin induces apoptotic lung cancer cell death via activation of DR4 pathway. Arch Pharm Res 37: 501-511.[Crossref]

40. Salma Y, Lafont E, Therville N, Carpentier S, Bonnafé MJ, et al. (2009) The natura marine anhydrophytosphingosine, Jaspine $\mathrm{B}$, induces apoptosis in melanoma cells by interfering with ceramide metabolism. Biol Pharm 78: 477-485.[Crossref]

Copyright: (C2016 Carvajal A. This is an open-access article distributed under the terms of the Creative Commons Attribution License, which permits unrestricted use, distribution, and reproduction in any medium, provided the original author and source are credited. 